\title{
Vapor Phase Sensing Using Metal Nanorod Thin Films Grown by Cryogenic Oblique Angle Deposition
}

\author{
Piyush Shah, ${ }^{1}$ Dongquan Ju, ${ }^{2}$ Xiaoxu Niu, ${ }^{3}$ and Andrew M. Sarangan' \\ ${ }^{1}$ Electro-Optics Graduate Program, University of Dayton, 300 College Park, Dayton, OH 45469-2951, USA \\ ${ }^{2}$ Department of Physics, Harbin Institute of Technology, Harbin 150001, China \\ ${ }^{3}$ Graduate Materials Engineering Program, University of Dayton, 300 College Park, Dayton, OH 45469-2951, USA
}

Correspondence should be addressed to Andrew M. Sarangan; sarangan@udayton.edu

Received 3 September 2013; Accepted 29 October 2013

Academic Editor: Matteo Tonezzer

Copyright (C) 2013 Piyush Shah et al. This is an open access article distributed under the Creative Commons Attribution License, which permits unrestricted use, distribution, and reproduction in any medium, provided the original work is properly cited.

\begin{abstract}
We demonstrate the chemical sensing capability of silver nanostructured films grown by cryogenic oblique angle deposition (OAD). For comparison, the films are grown side by side at cryogenic $(\sim 100 \mathrm{~K})$ and at room temperature $(\sim 300 \mathrm{~K})$ by e-beam evaporation. Based on the observed structural differences, it was hypothesized that the cryogenic OAD silver films should show an increased surface enhanced Raman scattering (SERS) sensitivity. COMSOL simulation results are presented to validate this hypothesis. Experimental SERS results of 4-aminobenzenethiol (4-ABT) Raman test probe molecules in vapor phase show good agreement with the simulation and indicate promising SERS applications for these nanostructured thin films.
\end{abstract}

\section{Introduction}

The detection and identification of hazardous chemical and biological agents is important for several areas of defense and security as well as in other industries that deal with hazardous chemicals [1]. Gas chromatography has the advantage of providing quick and accurate detection capability; however cost, size, and lack of portability have limited its widespread use [2]. Ion-mobility spectrometer is another popular technique for chemical sensing; however the level of information that can be extracted is not comparable to most vibrational spectroscopy techniques [3]. Surface enhanced Raman scattering (SERS) has shown the ability to detect the presence of very low concentrations of chemical agents quickly $[1,4-6]$. Several groups have also demonstrated a portable Raman setup for chemical and biosensing applications $[7,8]$. In many of these applications, colloidal silver nanoparticles are used as the SERS substrates, which limits these to only liquid phase applications [9]. On the other hand, silver nanostructured SERS substrates have the flexibility to work with liquids or vapors. Some of the earlier literature on SERSbased vapor sensing includes simulants for highly toxic chemicals such as nerve and mustard agents [7]. Most of these used electrochemically roughened silver substrates or silver film over nanosphere substrates. All of these techniques are limited by the available surface area for the vapor molecules to bind and adsorb onto. On the other hand, nanorod-based substrates can offer a significantly greater surface area for the same foot print area.

Oblique angle physical vapor deposition technique has led to the evolution of a new class of thin films with very large effective surface areas. The technique is based on atomistic level self-shadowing. Whereas in typical thin film deposition setups the substrate is held normal to the vapor source, in the OAD setup the substrate is held at a very large oblique angle with respect to the incoming vapor flux. This angle creates shadows behind each condensing atom preventing subsequent atoms from condensing in the shadowed areas. Instead, they land on the previously nucleated sites resulting in the evolution of columnar morphology. A detailed overview and potential applications of these thin films are given in the review paper by Hawkeye and Brett [10]. Even though OAD has been demonstrated to work well with metals like titanium (Ti), chromium $(\mathrm{Cr})$, nickel $(\mathrm{Ni})$, and so forth, it has shown limited success with soft metals like silver $(\mathrm{Ag})$, gold $(\mathrm{Au})$, and copper $(\mathrm{Cu})[11,12]$. Most of the results with the latter 
metals show lower aspect ratios and/or collapsed columns, especially when the substrate is held at ambient temperatures during the growth.

In this study we show the results of silver nanorod (AgNR) thin films grown by OAD at cryogenic temperatures $(\sim 100 \mathrm{~K})$ compared to room temperature $(\sim 300 \mathrm{~K})$. The AgNR substrates were then incubated with vapors of 4-ABT and characterized for SERS measurements. Experimental results show a significant improvement in vapor phase detection capability of the cryogenically grown films compared to the room temperature grown films. These results are also confirmed by COMSOL MultiPhysics simulations of the plasmonic field enhancement in the AgNR.

\section{Experimental Details}

2.1. Fabrication. All of our depositions were done using an MDC evap-4000 e-beam evaporator with a throw distance of approximately $0.5 \mathrm{~m}$. A substrate mounting plate was designed and built to carry liquid nitrogen in an open-cycle Dewar configuration. The samples were held by angled blocks made from $\mathrm{Cu}$ and Teflon with a preset angle of $88^{\circ}$ with respect to the incident flux and mounted to this cold plate. This allows concurrent growth of a cryogenically cooled sample and an ambient temperature sample on the $\mathrm{Cu}$ and Teflon blocks, respectively. Separate thermocouples were attached to the blocks to monitor the substrate temperatures. During growth, the $\mathrm{Cu}$ block was at approximately $100 \mathrm{~K}$ and the Teflon block was close to $300 \mathrm{~K}$. Further details of this experimental setup are given in [13]. The substrates used for all the experiments were $\langle 100\rangle$ silicon and the films were grown at a rate of $3 \AA / \mathrm{s}$. The film thicknesses were measured using a quartz crystal microbalance at normal incidence (i.e., not at a glancing angle).

\subsection{Vapor Phase Deposition of 4-Aminobenzenethiol (4-ABT)}

Test Probes. 4-ABT molecules contain sulfur which binds very effectively with the silver surface. The compound is highly volatile due to the presence of thiol and amino groups. In the setup shown in Figure 1, approximately 10 milligrams powder of 4-ABT was loaded in a $10 \mathrm{~mL}$ glass beaker. A microscope glass slide with double sided tape was used to attach the AgNRs samples. A plain untreated silicon sample was also incubated with the 4-ABT vapor as the background control sample. The samples were incubated for different time periods to vary the concentration of the vapor being deposited on the substrate. Due to the sensitivity of the 4-ABT molecule to the ambient moisture, the incubation was done in a glove box setup, purged three times with high purity $\mathrm{N}_{2}$ prior to allowing the evaporation to occur. The samples were exposed to the 4-ABT vapor for different times starting with 60 min exposure followed by 30,20 , and 5 min exposure.

2.3. Micro-Raman and SERS Measurements. The SERS measurements were obtained using a Raman measurement tool (LabRamHR 800 system). A $632.8 \mathrm{~nm}$ HeNe laser, with an unattenuated output power of $15 \mathrm{~mW}$, was used as the excitation source. The backscattered Raman and SERS signals were

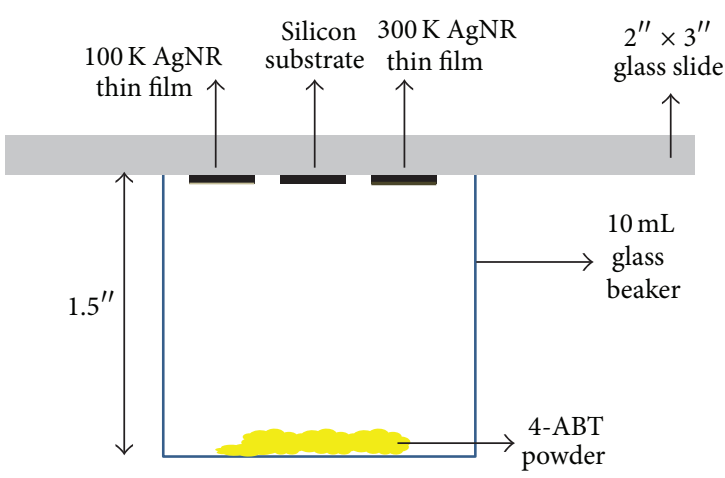

FIGURE 1: Schematic showing experimental setup used for $\mathrm{Ag}$ nanorods thin films incubation with 4 -ABT vapors.

collected using a thermoelectrically cooled CCD detector. The focused laser beam spot size on the AgNR surface was about $1 \mu \mathrm{m}$. Under these experimental conditions, the spectral resolution was about $1 \mathrm{~cm}^{-1}$. Point measurements were conducted to determine the appropriate integration times, and optical density filters were used to reduce the incident laser power. A $5 \mathrm{~s}$ acquisition time and signal averaging over 3 cycles were used to acquire the spectra with adequate signal to noise ratio. SERS spectra at random locations were collected across the AgNR and the silicon substrates.

\section{Results and Discussion}

3.1. SEM Results. Figure 2 shows the SEM cross section views of the AgNR thin films grown at $100 \mathrm{~K}$ and $300 \mathrm{~K}$ substrate temperatures. The distinct difference in the morphology between the two films can be immediately seen from these images. Table 1 shows quantitative differences between the two AgNRs. The AgNRs grown at $100 \mathrm{~K}$ was $28 \%$ smaller in diameter and $56 \%$ longer compared to the $300 \mathrm{~K}$ sample, resulting in an overall increase in their aspect ratios. The $100 \mathrm{~K}$ AgNRs also shows a curvature, or sag, in its structure, which we have also observed in other soft metals (and not observed in metals like $\mathrm{Cr}, \mathrm{Ni}$, and $\mathrm{Ti}$ ). The $100 \mathrm{~K}$ AgNRs also shows a $39 \%$ increase in the rod density per unit substrate area. All of these morphological differences in the $100 \mathrm{~K}$ AgNRs effectively increase the surface area available for molecular adsorption. This change in morphology is attributable to the reduction in surface diffusion and mobility of the silver atoms at cryogenic temperatures. At cryogenic temperatures, the atoms freeze on impact at the landing site. This results in very small clusters of atoms on which the columns evolve. At higher temperatures, atoms have enough energy to migrate some distance away from their landing sites resulting in larger nucleation clusters. Even after the column growth has begun, higher growth temperatures can induce a greater atomic diffusion on the top of each column. This effect is often observed as an enlargement of the column diameter with increasing height, like a mushroom structure. The increase in surface area of the $100 \mathrm{~K}$ grown sample was estimated to be about $214 \%$ compared to room temperature 
TABLE 1: Morphological comparison of silver nanorods thin films grown at $100 \mathrm{~K}$ and $300 \mathrm{~K}$.

\begin{tabular}{lcc}
\hline & \multicolumn{2}{c}{$\mathrm{Ag}$} \\
\hline Substrate temperature $(\mathrm{K})$ & 100 & 300 \\
Length $(\mathrm{nm})$ & $428 \pm 20$ & $273 \pm 21$ \\
Diameter $(\mathrm{nm})$ & $36 \pm 10$ & $50 \pm 3$ \\
Tilt angle $\left(^{\circ}\right)$ & $43 \pm 10$ & $18 \pm 1$ \\
Nanorods density $\left(\# / \mu \mathrm{m}^{2}\right)$ & $53 \pm 5$ & $38 \pm 4$ \\
\hline
\end{tabular}

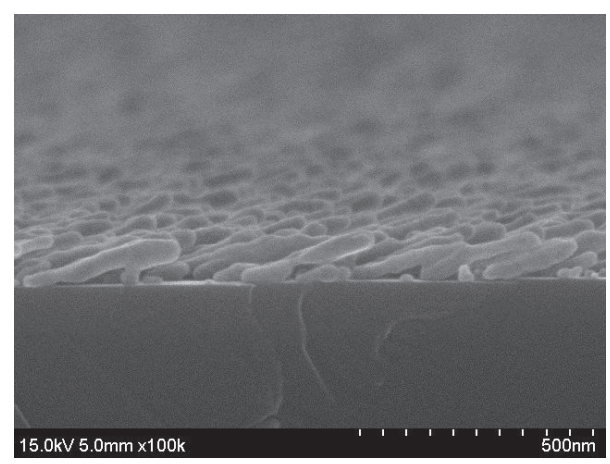

(a)

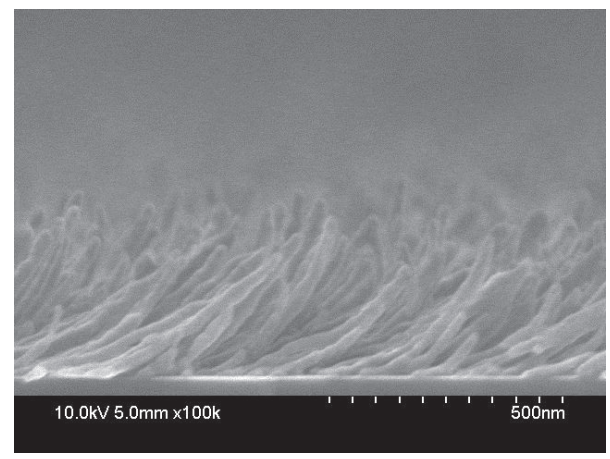

(b)

Figure 2: Side view of SEM results of (a) AgNRs at $300 \mathrm{~K}$ and (b) AgNRs at $100 \mathrm{~K}$.

sample. Further experimental results of the temperature effects can be found in [13].

3.2. COMSOL MultiPhysics Modelling. For computing the SERS enhancement factor a three-dimensional full wave simulation using COMSOL MultiPhysics was performed. In the simulation, one unit cell which consists of one individual nanorod is modeled by setting a periodic boundary condition. $633 \mathrm{~nm}$ wavelength was used as the excitation light source, which is the same as the one used in the subsequent experimental SERS measurements. The optical properties of silver were taken from the experiment data of Johnson and Christy [14]. The normal incident plane wave has the polarization direction along the $L_{x}$ direction of the nanorod array as shown in Figure 3.

Figure 4 shows normalized electric field distribution of the $100 \mathrm{~K}$ and $300 \mathrm{~K}$ AgNRs. The scale bar on the right represents the normalized amplitude of the scattered electric

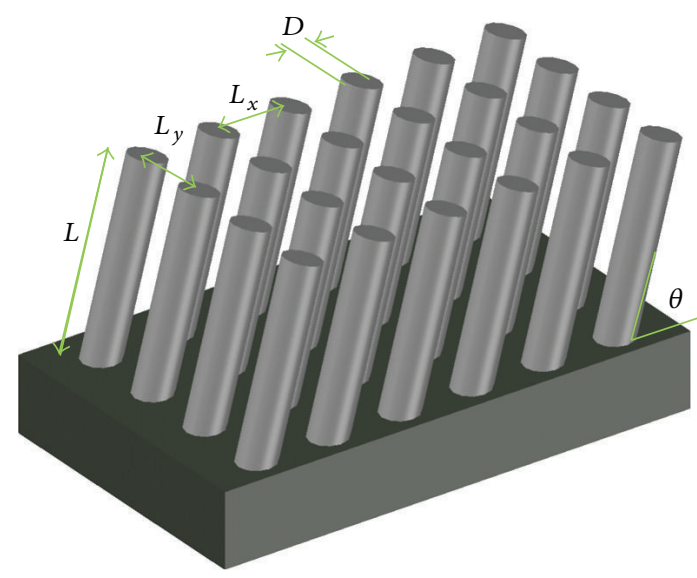

FIGURE 3: 3D schematic showing physical parameters of silver nanorods used in the simulation.

field with respect to the incident electric field amplitude. The interaction between the nanostructured metallic surface and the incident light source generates a localized surface plasmon resonance (LSPR) [15]. This causes an enhancement in the electric field distribution along the nanorod surface for both $100 \mathrm{~K}$ and $300 \mathrm{~K}$ AgNRs. As seen in Figure 4(c), the LSPR forms a standing wave along each nanorod $[16,17]$. Due to this standing wave, the hot spots were not only observed on the tip of individual nanorods but were also observed along the sidewalls of the nanorods.

Based on the electric field enhancement observed in the $100 \mathrm{~K}$ grown AgNRs compared to the $300 \mathrm{~K}$ AgNRs, a parametric study was done by varying the spatial distances $\left(L_{x}\right)$ between the adjacent nanorods for the $100 \mathrm{~K}$ configuration. Figure 5 shows the results of this parametric study. The change in spatial distance $L_{x}$ affects the plasmonic resonance between the two adjacent nanorods along the $x$ direction. This effect causes a change in the amplitude and frequency of the standing waves along the nanorod direction, which causes a change in the location and magnitude of the hot spots.

The electric field distribution along the nanorods surface was used to calculate the surface average SERS enhancement factor $\left(G_{\text {SERS }}\right)[18]$

$$
\begin{gathered}
G_{\text {SERS }}=\frac{\int g d s}{A_{0}}, \\
g=\frac{\left|E_{\text {pump }}\right|^{2}\left|E_{\text {stokes }}\right|^{2}}{\left|E_{0}\right|^{4}} \approx \frac{\left|E_{\text {pump }}\right|^{4}}{\left|E_{0}\right|^{4}}
\end{gathered}
$$

where $\left|E_{\text {pump }}\right|^{2}\left|E_{\text {stokes }}\right|^{2} \approx\left|E_{\text {pump }}\right|^{4}$ and $E_{\text {stokes }}, E_{\text {pumps }}$, and $E_{0}$ are the enhanced Raman, enhanced incident electric field, and the incident electric field, respectively. $g$ is the localized SERS enhanced factor. $A_{0}$ is the corresponding unstructured area $\left(L_{x} \times L_{y}\right)$. The calculated enhancement factor for the $100 \mathrm{~K}$ grown AgNRs was $G_{\text {SERS }}=1.85 \times 10^{6}$ and that of $300 \mathrm{~K}$ grown AgNRs was $G_{\text {SERS }}=6.22 \times 10^{5}$. Hence the enhancement factor is larger by approximately a factor of 3 for the $100 \mathrm{~K}$ sample compared to the $300 \mathrm{~K}$ sample. 


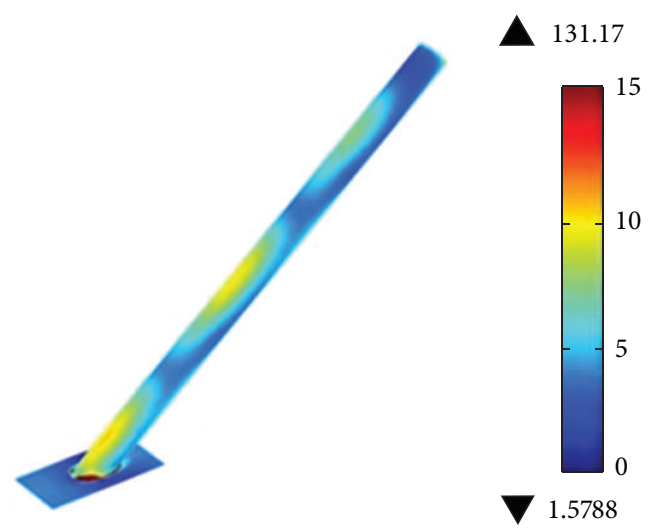

(a)

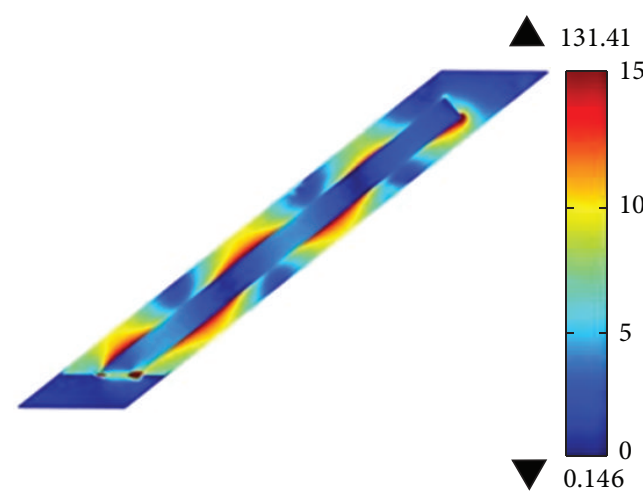

(c)

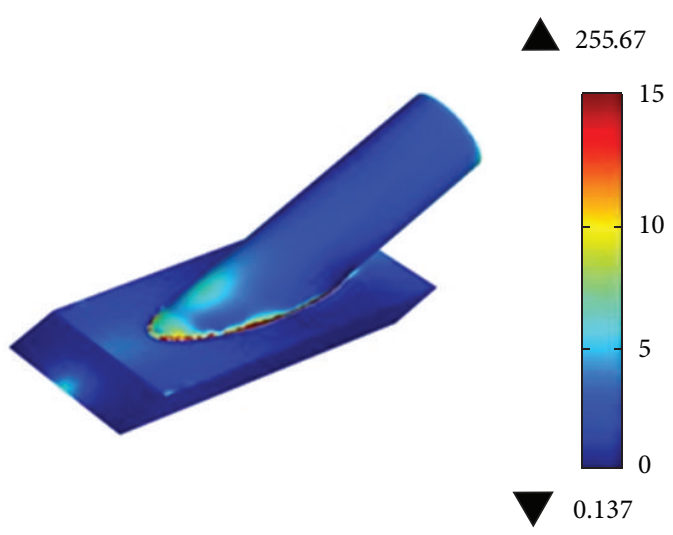

(b)

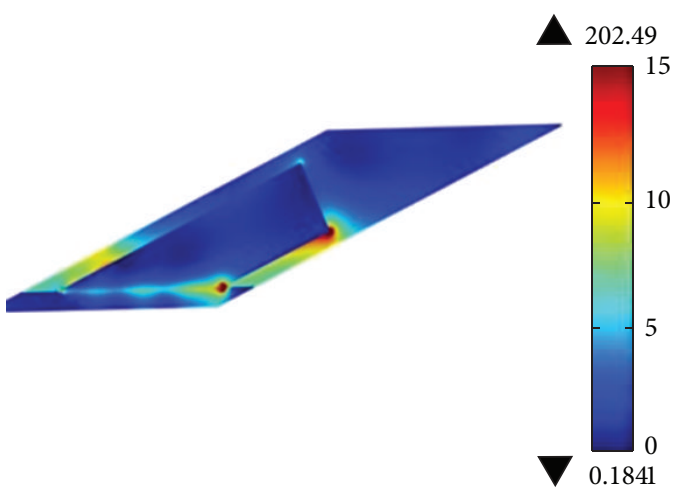

(d)

FIGURE 4: 3D normalized electric field distributions of nanorod grown at (a) $100 \mathrm{~K}$ and (b) $300 \mathrm{~K}$; 2D electric field distributions of nanorod grown at (c) $100 \mathrm{~K}$ and (d) $300 \mathrm{~K}$.

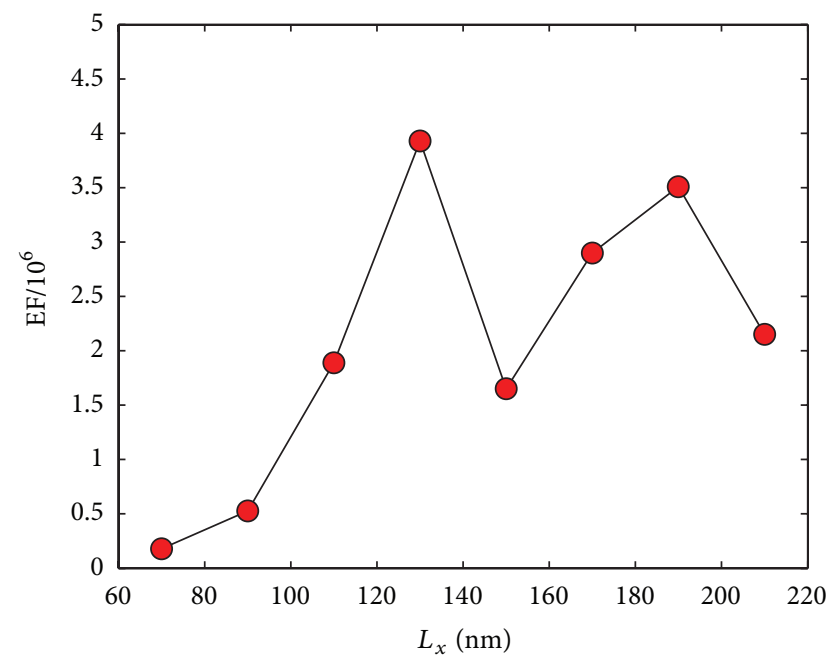

FIGURE 5: SERS enhancement factor as a function of different spatial distances $\left(L_{x}\right)$ between the adjacent nanorods grown at $100 \mathrm{~K}$.

3.3. SERS Measurement Results. Figure 6 shows the SERS spectra collected from $100 \mathrm{~K}$ and $300 \mathrm{~K}$ AgNRs that were incubated with 4 -ABT vapor for 5, 20,30, and 60 minutes. It is clear that 4-ABT vapor was detected for all exposure times and the SERS signal intensities from $100 \mathrm{~K}$ AgNRs are significantly larger than the $300 \mathrm{~K}$ AgNRs. During the sample incubation with 4-ABT vapor, a plain silicon substrate was also incubated. Raman spectra from the silicon sample showed no detectable presence of 4-ABT molecules. The key difference between the AgNRs at $100 \mathrm{~K}$ and $300 \mathrm{~K}$ is the ability to resolve and detect all representative 4 -ABT peaks. For samples that were exposed for 30 and 60 minutes, it appears that the SERS spectra from $100 \mathrm{~K}$ AgNRs distinctly detect and resolve all 4ABT representative peaks. This behavior may be explained by the increase in nanostructured surface area available in the $100 \mathrm{~K}$ grown AgNRs compared to the $300 \mathrm{~K}$ sample. Also the increased plasmonic activity and electric field enhancement provides another cause for the increase in molecular detection sensitivity in the $100 \mathrm{~K}$ AgNRs compared to the $300 \mathrm{~K}$ AgNRs. The $300 \mathrm{~K}$ AgNRs have a collapsed physical structure as observed in Figure 2(b). This will prevent the molecules from being absorbed along the sidewall because less surface area is presented to the molecules. The SERS signal intensity from the 5-minute exposed sample appears higher and has shown a different baseline compared to the 20-, 30-, and 60minute exposure samples. This was presumably due to the 4-ABT molecules degradation or reactivity with the ambient prior to adsorption on the AgNRs surface. Nevertheless, it is 


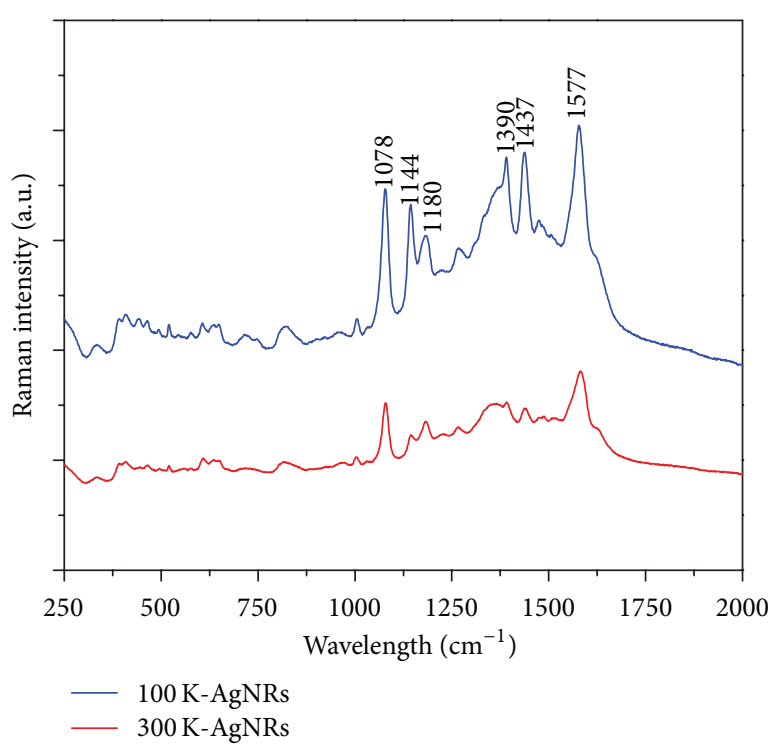

(a)

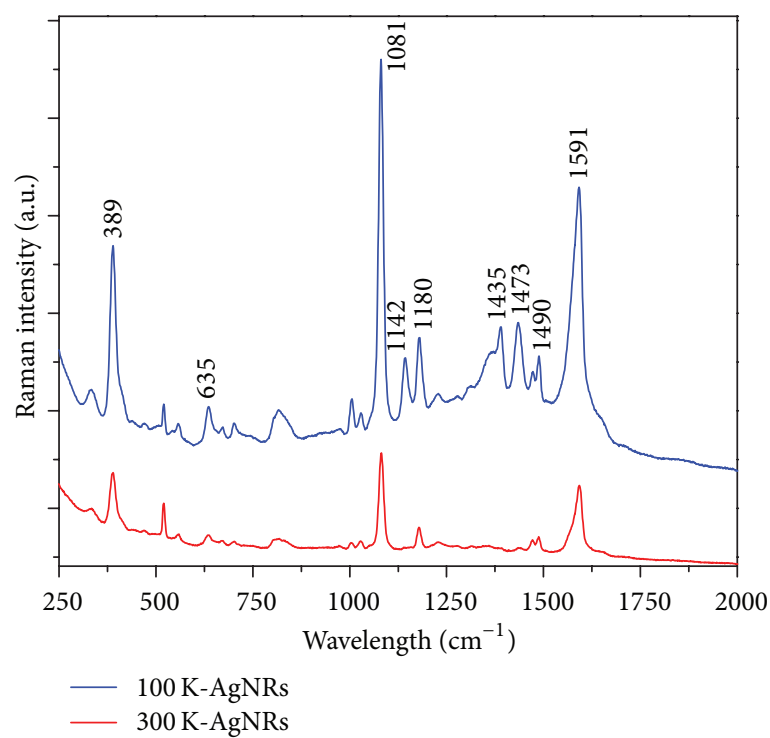

(c)

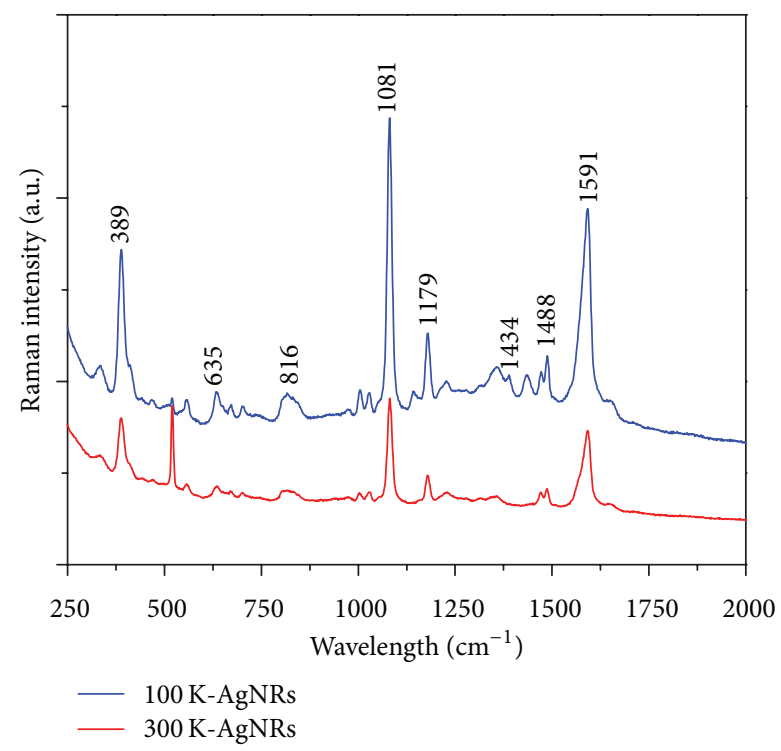

(b)

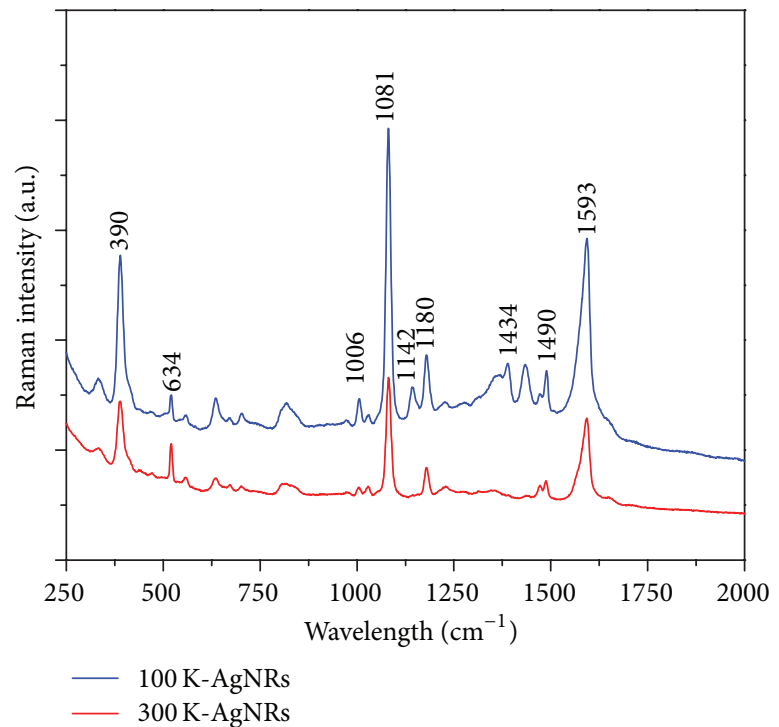

(d)

FIGURE 6: SERS response from $100 \mathrm{~K}$ (blue) and $300 \mathrm{~K}$ (red) grown AgNRs thin films after 4-ABT exposure with varying times (a) 5 min, (b) $20 \mathrm{~min}$, (c) $30 \mathrm{~min}$, and (d) $60 \mathrm{~min}$.

clear that even a 5-minute exposure to 4-ABT vapor has been detected with a significantly stronger SERS response with $100 \mathrm{~K}$ grown AgNRs compared to that of the $300 \mathrm{~K}$ sample. Based on the SERS response from 5-minute-exposed sample, it might be possible to improve the lower limit of detection by decreasing the exposure time even further and still be able to acquire SERS spectra with high signal to noise ratio. However, we have not explored this lower limit in this study.

SERS spectra from unexposed $100 \mathrm{~K}$ and $300 \mathrm{~K}$ samples were also acquired prior to incubation with 4-ABT vapor. These spectra typically have shown varied amounts of Raman peaks indicative of unidentified organics present on the surface of AgNRs. The intensity of these Raman peaks was higher on $100 \mathrm{~K}$ AgNRs samples compared to $300 \mathrm{~K}$ samples.
The two possible sources for these organics were the substrate cleaning using organic solvents (acetone, isopropyl alcohol, and methanol) prior to the growth and the exposure of samples to the ambient environment. However the level of these Raman peaks was buried in the baseline of the Raman spectra once the samples were exposed to 4-ABT. This indicates that the contribution of noise due to the unidentified organic peaks to the acquired 4-ABT spectra is practically negligible. Raman spectra from the silicon samples incubated concurrently with the AgNRs samples showed no evidence of 4-ABT molecules on the surface. The spectra showed a strong peak at $521 \mathrm{~cm}^{-1}$ indicative of the silicon substrate. Considering the minimal interference of background Raman spectra from AgNRs samples, $1080 \mathrm{~cm}^{-1}$ SERS peak was 
chosen as the representative peak to compare the integrated area intensities. An average of $282 \%$ improvement in SERS response was observed in $100 \mathrm{~K}$ grown AgNRs compared to that of $300 \mathrm{~K}$ grown AgNRs. In the present study, the AgNRbased nanostructured surface has shown high selectivity for detecting 4-ABT molecule even in the presence of low concentrations of organics and other impurities in the ambient environment. Furthermore, we have not examined the reusability of the substrates, but we speculate that it can potentially be done by heating the substrate to drive off the adsorbed molecules.

\section{Conclusions}

In summary, the improved morphology of Ag nanorods thin films grown at $100 \mathrm{~K}$ temperature has a strong influence on the enhanced plasmonic activity compared to room temperature grown samples. We have demonstrated this with 4-ABT test probe molecules and also validated the results with FEM simulation results. Although we only demonstrated this with 4-ABT molecules, any organic molecules that adsorb easily to the AgNRs surface and have distinct Raman spectra are effective candidates for detection using this nanostructured surface.

\section{Acknowledgments}

The authors would like to thank Adam Stahler and Ioana Pavel at Wright State University for help with SERS measurements.

\section{References}

[1] K. B. Biggs, J. P. Camden, J. N. Anker, and R. P. V. Duyne, "Surface-enhanced raman spectroscopy of benzenethiol adsorbed from the gas phase onto silver film over nanosphere surfaces: determination of the sticking probability and detection limit time," Journal of Physical Chemistry A, vol. 113, no. 16, pp. 4581-4586, 2009.

[2] P. A. Smith, D. Koch, G. L. Hook et al., "Detection of gasphase chemical warfare agents using field-portable gas chromatography-mass spectrometry systems: instrument and sampling strategy considerations," TrAC Trends in Analytical Chemistry, vol. 23, no. 4, pp. 296-306, 2004.

[3] D. C. Collins and M. L. Lee, "Developments in ion mobility spectrometry-mass spectrometry," Analytical and Bioanalytical Chemistry, vol. 372, no. 1, pp. 66-73, 2002.

[4] J. D. Driskell, S. Shanmukh, Y. Liu et al., "The use of aligned silver nanorod arrays prepared by oblique angle deposition as surface enhanced raman scattering substrates," Journal of Physical Chemistry C, vol. 112, no. 4, pp. 895-901, 2008.

[5] Y.-J. Liu, H. Y. Chu, and Y.-P. Zhao, "Silver nanorod array substrates fabricated by oblique angle deposition: morphological, optical, and SERS characterizations," Journal of Physical Chemistry C, vol. 114, no. 18, pp. 8176-8183, 2010.

[6] S. Shanmukh, L. Jones, J. Driskell, Y. Zhao, R. Dluhy, and R. A. Tripp, "Rapid and sensitive detection of respiratory virus molecular signatures using a silver nanorod array SERS substrate," Nano Letters, vol. 6, no. 11, pp. 2630-2636, 2006.
[7] D. A. Stuart, K. B. Biggs, and R. P. Van Duyne, "Surfaceenhanced Raman spectroscopy of half-mustard agent," Analyst, vol. 131, no. 4, pp. 568-572, 2006.

[8] H. Y. Chu, Y. Liu, Y. Huang, and Y. Zhao, "A high sensitive fiber SERS probe based on silver nanorod arrays," Optics Express, vol. 15, no. 19, pp. 12230-12239, 2007.

[9] I. E. Pavel, K. S. Alnajjar, J. L. Monahan et al., "Estimating the analytical and surface enhancement factors in SurfaceEnhanced Raman Scattering (SERS): a novel physical chemistry and nanotechnology laboratory experiment," Journal of Chemical Education, vol. 89, no. 2, pp. 286-290, 2012.

[10] M. M. Hawkeye and M. J. Brett, "Glancing angle deposition: fabrication, properties, and applications of micro- and nanostructured thin films," Journal of Vacuum Science and Technology A, vol. 25, no. 5, pp. 1317-1335, 2007.

[11] D. Schmidt, B. Booso, T. Hofmann, E. Schubert, A. Sarangan, and M. Schubert, "Monoclinic optical constants, birefringence, and dichroism of slanted titanium nanocolumns determined by generalized ellipsometry," Applied Physics Letters, vol. 94, no. 1, Article ID 011914, 2009.

[12] D. Schmidt, B. Booso, T. Hofmann, E. Schubert, A. Sarangan, and M. Schubert, "Generalized ellipsometry for monoclinic absorbing materials: determination of optical constants of $\mathrm{Cr}$ columnar thin films," Optics Letters, vol. 34, no. 7, pp. 992-994, 2009.

[13] P. J. Shah, X. Niu, and A. M. Sarangan, "High aspect ratio silver nanorod thin films grown at cryogenic temperature substrates," Journal of Nanoscience Letters, vol. 3, p. 19, 2012.

[14] P. B. Johnson and R. W. Christy, "Optical constants of the noble metals," Physical Review B, vol. 6, no. 12, pp. 4370-4379, 1972.

[15] K. Kneipp, H. Kneipp, I. Itzkan, R. R. Dasari, and M. S. Feld, "Surface-enhanced Raman scattering and biophysics," Journal of Physics Condensed Matter, vol. 14, no. 18, pp. R597-R624, 2002.

[16] D. P. Lyvers, J.-M. Moon, A. V. Kildishev, V. M. Shalaev, and A. Wei, "Gold nanorod arrays as plasmonic cavity resonators," ACS Nano, vol. 2, no. 12, pp. 2569-2576, 2008.

[17] J. Yao, X. Yang, X. Yin, G. Bartal, and X. Zhang, "Threedimensional nanometer-scale optical cavities of indefinite medium," Proceedings of the National Academy of Sciences of the United States of America, vol. 108, no. 28, pp. 11327-11331, 2011.

[18] P. L. Stiles, J. A. Dieringer, N. C. Shah, and R. P. Van Duyne, "Surface-enhanced Raman spectroscopy," Annual Review of Analytical Chemistry, vol. 1, no. 1, pp. 601-626, 2008. 

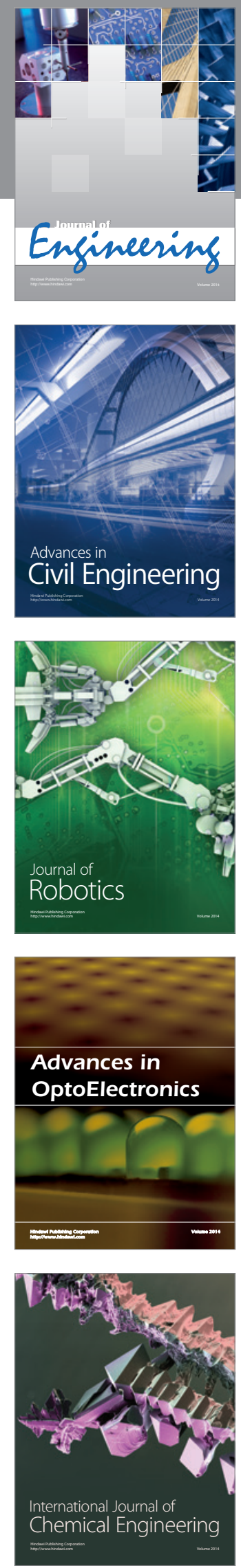

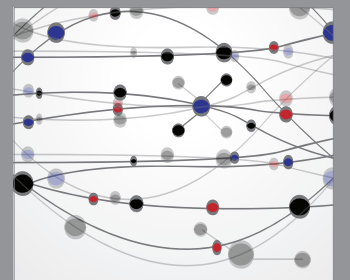

The Scientific World Journal
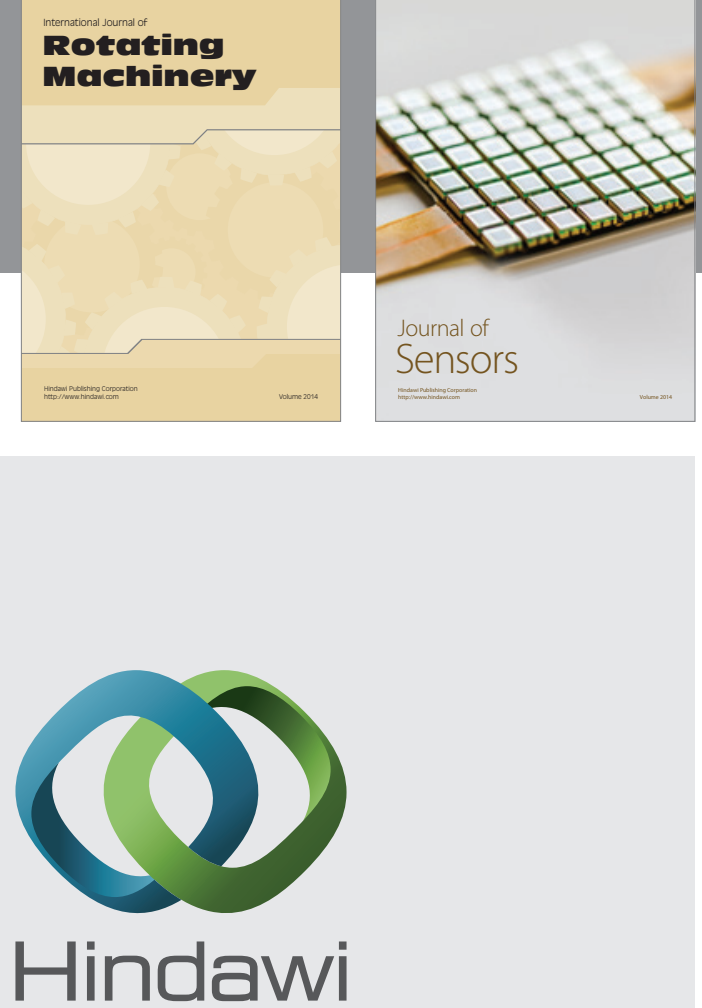

Submit your manuscripts at http://www.hindawi.com
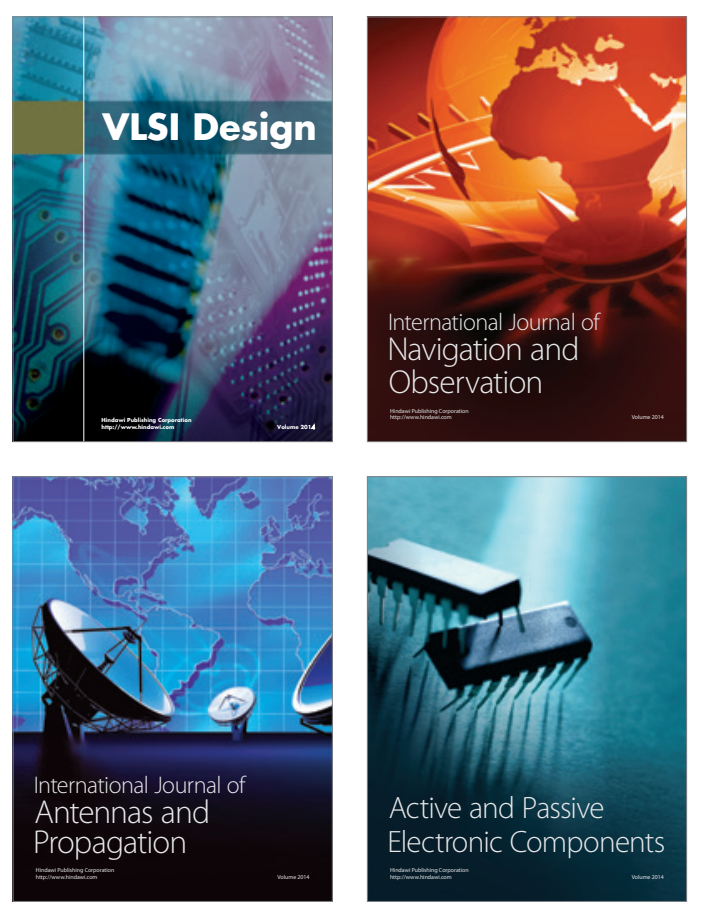
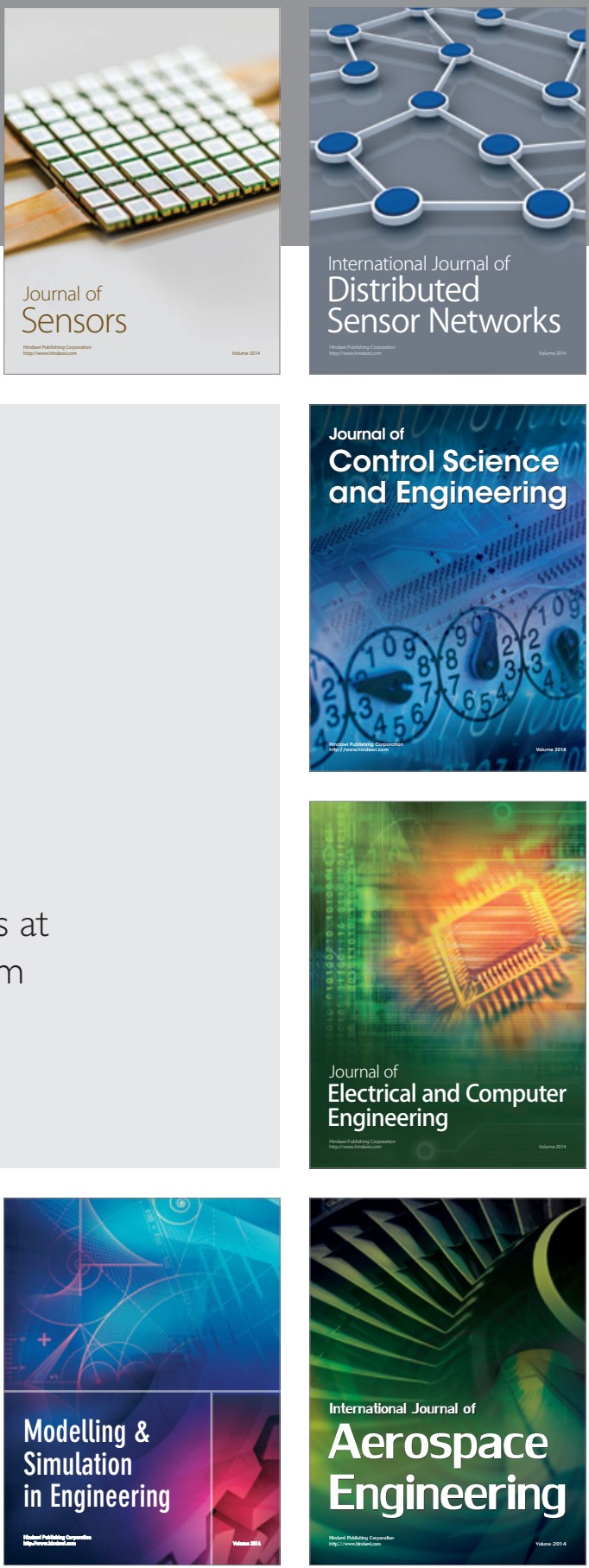

Journal of

Control Science

and Engineering
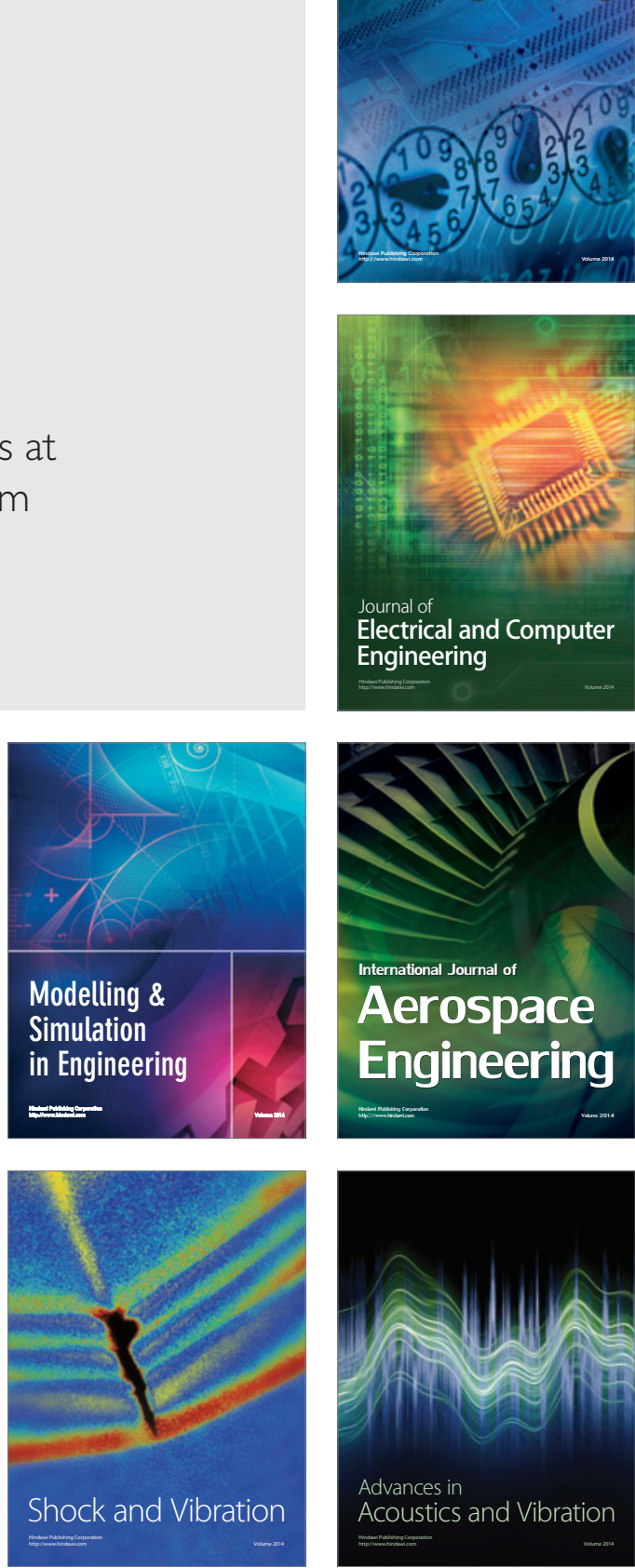